\title{
Entre gestion de crise et vigilance : quand les médias et l'administration publique sont appelés à construire un référentiel partagé
}

Catherine Fallon et Geoffrey Joris

\section{OpenEdition}

\section{Journals}

Édition électronique

URL : http://journals.openedition.org/communicationorganisation/709

DOI : 10.4000/communicationorganisation.709

ISSN : 1775-3546

Éditeur

Presses universitaires de Bordeaux

Édition imprimée

Date de publication : 1 juin 2009

Pagination : 60-69

ISSN : 1168-5549

Référence électronique

Catherine Fallon et Geoffrey Joris, « Entre gestion de crise et vigilance : quand les médias et l'administration publique sont appelés à construire un référentiel partagé », Communication et organisation [En ligne], 35 | 2009, mis en ligne le 01 décembre 2012, consulté le 30 avril 2019. URL : http://journals.openedition.org/communicationorganisation/709 ; DOI : 10.4000/ communicationorganisation.709 
Dossier : Repenser la communication des organisations publiques

\begin{abstract}
Résumé
Les moments de crise sont riches d'enseignements quant aux problèmes de collaboration entre administrations et médias, car toute crise est d'abord une crise d'information. Les autorités se trouvent empêtrées dans un réseau d'obligations contradictoires, appelées à collaborer avec les médias, partenaires incontournables pour informer et cadrer la catastrophe. Si la communication de crise obéit à des contraintes particulières, elle se construit en prolongement des régimes « hors crise ». Il convient de prolonger l'analyse des régimes de communication de crise à la période de vigilance médiatique afin de cerner les contours et enjeux des régimes informationnels dans lesquels elle se replace.
\end{abstract}

\title{
Mots-clés
}

Administration publique, gestion de crise, médias, régime informationnel, Société du risque.

\begin{abstract}
Analysis of crisis management, often strongly mediatized, is particularly fruitful to unveil collaboration problems between media and public authorities as technological crisis are first and foremost information crisis. Authorities are tightened with regulatory and administrative constrains often contradictory to the logics of crisis communication, while media are unavoidable as partners to inform and enrol the public, amplifying and framing the accident. Crisis communication should be considered as rooted in "vigilance" communication. Analysis of crisis communication helps unveil the characteristics of such informational regimes.
\end{abstract}

Key-Words

Crisis management, information regime, media, Risk Society, public administration.

Catherine Fallon est ingénieur et sociologue. Elle est chercheur à l'Université de Liège en Science Politique, et est spécialisée dans l'évaluation de l'action publique pour la gestion des risques technologiques.

Geoffrey Joris est politologue et aspirant F.N.R.S. Il est basé à l'Université de Liège. Il prépare une thèse sur la transformation de la fonction publique en Belgique (gestion d'incertitude / gestion du changement). 


\section{Entre gestion de crise et vigilance : quand les médias et l'administration publique sont appelés à construire un référentiel partagé}

Catherine Fallon, Geoffrey Joris

\section{Catherine.Fallon@ulg.ac.be Joris.Geoffrey@ulg.ac.be}

Face aux enjeux d'une « Société du Risque » (Beck, 1992) fortement médiatisée, les administrations sont appelées à développer une politique de communication afin d'améliorer la visibilité de l'action publique et d'en assurer l'efficacité et l'efficience. Il s'agit de tisser les conditions d'un régime d'alliance entre médias et acteurs politiques, construit sur une structure de méfiance-confiance réciproque dont l'ambivalence explique la précarité.

Dans un tel contexte, l'irruption d'une crise engendre une rupture : elle impose aux autorités de déployer de nouvelles logiques d'action et elle ouvre de larges champs à l'information médiatique. Elle met sous tension les fragiles rapports de confiance entre acteurs politiques, administrations et médias. Mais elle se construit en prolongement des régimes de communication « hors crise », lesquels peuvent contribuer, dans un logique de vigilance, à jeter les bases d'un référentiel de sécurité (Brunet, 2007). À partir de l'expérience d'acteurs publics récoltée dans le cadre d'une après-midi d'étude portant sur l'analyse de communications en période de crise $^{76}$, cet article propose une stratégie d'analyse des modalités de gestion de la communication par les autorités aux différents moments d'une crise.

\section{Le régime informationnel de la «Société du Risque »}

Le concept de régime informationnel suppose la reconnaissance de l'existence d'interactions systémiques entre les structures sociales, économiques, politiques et informationnelles d'une société donnée. Ce système d'adaptation mutuelle et de co-construction des acteurs, de facto interdépendants, produit des ajustements spécifiques en fonction du domaine considéré et du contexte (Bimber, 2006). Le régime

\footnotetext{
${ }^{76}$ Cet article exploite les discussions d'un séminaire organisé à l'Université de Liège le 23 mai 2008 sur l'analyse de partenariat de crise entre administrations et médias: gestion de crises agro-alimentaires en Belgique, naufrage du Prestige en Espagne, accident de Tokaimura au Japon, accident de Ghislenghien : http://www.spiral.ulg.ac.be/medias_gestion_de_crise_08/
} 
informationnel se caractérise alors par un certain nombre d'éléments contextuels et sociaux. Dans le cas de l'information publique, il associe les autorités, les médias et les acteurs sociaux au sein d'un « spectacle politico-médiatique » (Edelman, 1991).

Les structures du régime informationnel ont été bouleversées par les récentes transformations techniques et économiques des médias, favorisant concentration, spécialisation et concurrence. En renforçant le registre émotionnel, les médias cherchent à enrôler leur audience à travers une vision dramatique de la réalité, accentuant un biais alarmiste et négativiste (Cvetkovitch, 1992).

Une partie du public met à l'épreuve les informations transmises, qu'elles proviennent des autorités ou des médias. D'une part, les canaux interpersonnels conservent toute leur importance: les conversations contribuent à tisser un socle culturel qui détermine les structures cognitives que les individus peuvent mobiliser pour le traitement des informations médiatiques. D'autre part, Internet ouvre un accès immédiat à une multitude d'informations relevant de registres différents. L'analyse de mobilisations relatives à des conflits d'implantations d'antennes GSM montre que les habitants recherchent des informations indépendamment des centres de référence officiels, non pour objectiver le débat mais pour renforcer leur maîtrise personnelle de la réalité (Borraz, 2004). Forts de ces sources, ils se sentent habilités à agir sur l'évènement, forçant sa mise à l'agenda politique et sa problématisation médiatique.

Alors que les citoyens sont mieux formés et plus aptes à s'exprimer, les relais revendicatifs institutionnels sont plus bureaucratisés et moins efficaces. Les analystes y relèvent l'existence d'un décalage croissant entre d'un côté le monde vécu familier, riche en références symboliques et de l'autre côté les sous-systèmes institutionnels autonomisés et spécialisés (Giddens, 1990). Face à ce recul des corps intermédiaires, les journalistes jouent un rôle plus important dans la mobilisation des émotions, entre autres par la construction de scénarios riches de sens dans lesquels les auditeurs se reconnaissent et identifient ce qu'on attend d'eux. (Coombs, 2007; Seon-Kyoung, 2009). Cette appropriation médiatique de l'expression publique est renforcée par l'effritement des institutions normatives qui cadraient fortement les débats publics et qui facilitaient la lecture des événements. Les médias jouent alors de plus en plus un rôle déterminant dans les mécanismes de mise à l'agenda politique des problématiques sociales (Derville, 2005), mobilisant pour ce 
magistère un spectre d'émotions plus large par des mises en scène capables de favoriser empathie, identification et compassion.

Face à ce nouveau régime informationnel, les autorités se voient contraintes d'assurer l'intelligibilité de leur action et d'enrôler des acteurs de plus en plus nombreux (Callon, 2001). L'élaboration d'une société de l'abondance médiatique impose une reconfiguration de l'activité communicationnelle de l'organisation bureaucratique.

\section{La collaboration entre médias et administration}

Utilisant des registres différents, médias et administrations ont chacun développé un regard spécifique sur leur audience et ses attentes, pris chacun dans des réseaux de contraintes professionnelles spécifiques qui limitent leur liberté d'action. Les journalistes travaillent avec des perspectives temporelles courtes et des contraintes rédactionnelles strictes propres à leur audience. Les responsables administratifs sont souvent spécialisés et attachés aux procédures et aux méthodes.

Le rapport entre autorités et journalistes résulte d'un équilibre entre méfiance et confiance: méfiance car les journalistes mettent en pratique un contre-pouvoir, confiance parce que les mêmes journalistes doivent établir des relations avec les autorités pour rassembler les informations nécessaires à l'exercice de ce contrepouvoir. Certaines administrations favorisent une stratégie d'ouverture, mais d'autres soutiennent une culture du contrôle, prétextant de ce que la transparence risquerait de dévoiler des divergences et d'exposer les citoyens aux difficultés de certaines problématiques et mettrait en danger les rapports de force dans les espaces de décision (Holmes, 2009). Nombre de responsables politiques préfèrent canaliser les inputs médiatiques en établissant une culture de contrôle de l'information, pour offrir une vision uniforme et contrôlée, stabilité qui peut se révéler fragile en cas de crise.

\section{La « Crise » comme moment privilégié de communication}

Pendant les moments de crise, les administrations doivent prendre des décisions urgentes alors que les faits sont incertains et les médias particulièrement présents. La crise déstabilise les moyens d'action et ouvre le champ à l'attention médiatique (Lagadec, 1991). Elle menace le fonctionnement du système lui-même dont elle ébranle les références essentielles et les choix collectifs des acteurs affectés.

Dans ce contexte incertain, où trouver une source d'information fiable? Les sondages montrent que le public fait surtout confiance aux 
scientifiques, aux médecins, voire aux associations, mais beaucoup moins aux responsables politiques ou aux journalistes (Eurobaromètre, 2007). Et pourtant la responsabilité première de la communication de crise repose bien sur les autorités publiques, les élus et les canaux médiatiques. L'autorité publique endosse la responsabilité ultime en cette matière, tout en étant par ailleurs encastrée dans un réseau d'obligations en partie contradictoires : protection de la vie privée ; secret médical ou professionnel; protection de l'ordre public; prévention de faits punissables. Plutôt que de considérer la communication comme un processus d'interactions, l'administration tend à réduire sa mission à une simple transmission de faits, limitant ainsi les possibilités de mobilisation d'autres registres qui s'avéreraient plus efficaces (Holladay, 2009).

Les médias amplifient et dramatisent le contexte paroxystique de crise : ils cadrent la catastrophe et de ce fait modifient la gestion de la crise. De plus en plus rapides, ils exercent une forte pression pour obtenir des informations alors qu'elles sont encore très rares. Face à ces alliés incontournables que sont les médias, les autorités voudraient établir un certain contrôle au sein du régime d'information mais ils peinent à définir les modalités d'un partenariat équilibré. Les journalistes ont accès à de nombreuses sources d'information, auprès de la population, des autres services publics, des experts, etc. Ils jouent un rôle de premier plan, sélectionnant certaines dimensions de l'évènement et assurant un cadrage particulier pour la problématique, en fonction de la sensibilité de leur audience spécifique.

L'administration reste trop souvent étrangère au monde des médias auxquels elle est confrontée seulement aux moments de crise, quand la réalité échappe à son cadre de référence technico-rationnel. Les journalistes, pour leur part, sont davantage accoutumés aux périodes de crise dans la mesure où leurs conditions de travail les soumettent à des contraintes de rapidité, de délai, de choix de registre, etc. Il existe pourtant des expériences (Godard, 2002) de gestion intégrée des risques où les autorités développent en « temps de paix » des réseaux de communication dans le but d'emporter l'adhésion des experts, responsables politiques et du grand public, réseaux qui peuvent être rapidement mobilisés en cas de crise. Les relations médias administration se dessineront différemment pendant les trois moments de la crise : les périodes de prévention ou de vigilance, de l'intervention ou de crise, ou de l'après crise ou de la récupération (et $\mathrm{du}$ retour d'expérience). Dans une approche de communication stratégique, ces trois moments sont à la fois distingués et intégrés dans des démarches de communication spécifiques (Frosh, 2009). 
Entre gestion de crise et vigilance...

\section{Avant la crise : une collaboration de vigilance}

En dehors de la crise, les responsables administratifs et politiques ont tendance à se réserver l'espace d'expertise et de construction des savoirs. Mais la politique de communication externe a depuis peu acquis une valeur intrinsèque suite à diverses contraintes: droit à l'information, obligation de rendre des comptes, intelligibilité de l'action publique. La communication est dès lors considérée comme un instrument capable d'améliorer la qualité et l'efficacité de l'action publique.

Par exemple, une agence de sécurité de la chaîne alimentaire a pour mission d'assurer la construction d'un référentiel de sécurité partagé par tous les stakeholders. Le référentiel de «vigilance» devient luimême un produit de la communication du risque, car celle-ci produit du sens et contribue à cadrer les perceptions individuelles et sociales (Hupet, 2001). On retrouve ici un concept central de l'évaluation des politiques publiques (Muller, 2005), lequel considère l'action publique comme le résultat d'interactions entre de nombreux acteurs qui confrontent leurs visions du monde et leurs logiques d'action, pour définir des modes d'action communs, des représentations du réel qui vont orienter les choix et les actions afin de résoudre des problématiques reconnues communes.

Les systèmes d'alerte sont autant de moments d'apprentissage qui permettent à l'administration et aux médias de développer un référentiel commun de vigilance (Muller, 2005 ; Surel, 2000) pour informer et assurer un renforcement de la vigilance, tout en évitant les paniques et en relativisant les risques. La stratégie de communication externe développée par une administration pour renforcer la visibilité et le niveau de confiance du public permet d'établir les bases d'un partenariat avec les médias. En effet les journalistes sont appelés à traiter des questions techniques de plus en plus complexes et ils ont tendance à développer un certain rapport de confiance vis-à-vis d'organismes qui font preuve de transparence et d'ouverture.

\section{Activer un partenariat de crise}

En cas de crise, les procédures établies au préalable définissent un plan d'action, des protocoles et des responsables. La structure de communication définie dans les plans d'urgence se réduit encore souvent à un accès formel et unique, protocole utopique car le régime informationnel échappe alors à tout contrôle. 
Le public recourt de plus en plus aux nouveaux moyens de communication, GSM ou Internet, démultipliant les sources d'information et construisant des réseaux de collecte et de diffusion très réactifs, indépendamment des autorités et des médias officiels.

L'augmentation de la consommation de médias pendant les périodes de crise collective montre que le public cherche des informations et reconnaît l'importance du communicateur. Il est demandé aux responsables politiques d'occuper l'espace public, de donner du sens à la crise et à l'action collective. À défaut, les acteurs exposés aux risques et à leurs conséquences peuvent développer une méfiance à l'égard de ceux dont ils attendent une information qui leur permettrait de faire des choix informés. L'efficacité des décisions des autorités dépend en grande partie de leur pénétration sociale. Un silence ou une erreur peut être interprété comme une absence de co-orientation entre le responsable politique et ceux dont il a pour mission d'assurer la sécurité. Le processus de communication devient particulièrement instable quand les individus dont on sollicite l'adhésion condamnent une stratégie publique parce que cette option heurte leur représentation du juste (Slovic, 1993).

Les médias ne sont pas de simples canaux de communication: ils contribuent à construire le réel, créant auprès du public les conditions de représentation de la problématique par un processus de cadrage. Dans le cas de la marée noire du Prestige, les médias nationaux ont opéré une double expansion : ils présentaient la pire des zones sinistrées comme image représentative de l'ensemble de la zone sinistrée et celle-ci comme représentative de l'ensemble de la région, construisant un paysage désolant et générant involontairement un mouvement de mobilisation massive et un afflux de volontaires qui compliqua la tâche des gestionnaires. Pour leur part, les riverains étaient fascinés par les informations présentées par les médias, davantage que par que celles qu'ils pouvaient collecter eux-mêmes : la représentation médiatique de la réalité engendrait plus d'attraction que la réalité elle-même. Les habitants subissaient dès lors de plein fouet les biais médiatiques : négativisme, alarmisme et amplification.

\section{Après la crise : une logique commune d'apprentissage}

Après la crise, deux types de discours émergent: celui de la justification et celui de l'apprentissage. Il s'agit de répondre aux accusations du public et de restaurer une légitimité écornée par la crise, mais aussi d'en intégrer les leçons. Ces deux dynamiques associent des acteurs très différents. L'apprentissage nécessite une 
coopération externe sur plusieurs niveaux, avec les riverains et les autorités, créant des espaces de paroles et de circulation des informations, voire de contrôle des rumeurs. Les médias sont rarement associés aux retours d'expérience, car l'exposition publique de certains faits pourrait engager des actions en responsabilité. Par contre le registre de la justification transforme le traitement « post-crise » en moment privilégié de consolidation de nouvelles structures de représentations des problématiques, au sens de ce que Gamson (1989) dénomme un «media package », représentation dotée d'une cohérence interne forte qui propose aux spectateurs un cadrage particulier des évènements et leur assigne un sens. Rarement dévoilées ou même reconnues comme construction, riches de symboles et de choix normatifs, ces représentations structurent la vision du monde présentée par les journalistes et définissent les limites des conditions d'intervention. L'adoption de nouveaux « media packages » n'est pas instantanée et certains concepts mettent des années à s'imposer, comme ce fut le cas pour le "développement durable ». Leur construction porte la marque de ces moments de crise et des formes stabilisées dont les médias en habillent la mémoire. Le traitement médiatique construit un morceau du spectacle, limitant l'éventail des possibles, distribuant les rôles des acteurs, héros ou ennemis, choisissant et stabilisant des images autant que des discours chargés d'émotion et de passion (Gilpin, 2008).

\section{Conclusion}

L'analyse des crises contribue à mettre à jour les limites des processus de communication interne et externe initiés dans les périodes de vigilance (Brunet, 2007). Elle permet de proposer aux organisations publiques des pistes d'action pour créer les conditions de possibilité d'un partenariat avec les médias tenant compte des divergences de leurs contraintes et de leurs logiques de fonctionnement.

Autorités et médias doivent définir leurs objectifs et leur conception de l'information : qui informer ? Pourquoi ? Avec quels moyens ? Ce questionnement stratégique amène à construire un cadre bien charpenté quant aux matières à couvrir et aux manières d'aborder la communication. Mieux qu'un mythe partagé d'objectivité, il s'agit de reconnaitre les objectifs propres de chaque partenaire. Au-delà de l'information, la communication met en scène des faits au profit d'une vision particulière de la réalité et des évènements par un processus de problématisation, d'autant plus sensible que le régime informationnel de nos sociétés contemporaines se traduit par une restructuration du spectacle politique sous le mot d'ordre émotionnel. 
Les pratiques médiatiques sont des contraintes pour l'action publique, car le monde construit à travers les médias rend possibles certaines interventions mais pas d'autres. Identifier à qui bénéficie le problème, ainsi que les moyens mis en œuvre et les porteurs de projet, mettre en évidence les sources d'autorités et leurs objectifs contribuent à en dévoiler l'enracinement social. L'analyse des processus de communication pendant une crise permet de dévoiler les caractéristiques du régime informationnel à un moment où l'asymétrie entre autorités et médias est la plus faible, où la question de l'accès aux ressources est exacerbée. A partir d'une telle démarche critique, il est possible de définir, dans une logique plus normative, les termes d'une coopération adaptée aux intentionnalités des partenaires, à leurs contraintes professionnelles de qualité et d'efficacité, sans négliger le travail sur les représentations, pour fonder une action coopérative de communication à laquelle le public puisse être pleinement associé.

\section{Bibliographie}

BECK U., Risk society, Sage Publications, London, 1992.

BIMBER B., How information shapes political institutions, in Graber D., Media power in politics, CQPress, Washington, 2006, p. 8-18.

BORRAZ O., DEVIGNE M., SALOMON D., Controverses et mobilisations autour des antennes relais de téléphonie mobile, CSO, Paris, 2004.

BRUNET S., HOUBAERT P. Involving Stakeholders: the Belgian Fowl Pest Crisis, Journal of Risk Research, 2007, vol.10, p. 643-660.

CALLON M., LASCOUMES P., BARTHE Y., Agir dans un monde incertain. Essai sur la démocratie technique, Seuil, Paris, 2001.

COOMBS W.T., HOLLADY S.J., "The negative communication dynamic: Exploring the impact of stakeholder affect on behavioral intentions", Journal of Communication management, 2007, 11/4, p. 300-312.

CVETKOVITCH G., EARLE C. T., "Environmental hazards and the public", Journal of Social Issues, 1992, Vol. 48, n4, p.1-20.

DERVILLE G., Le pouvoir des médias : mythes et réalités, PUG, Coll. Politique « En plus », Grenoble, 2005.

EDELMAN M., Pièces et règles du jeu politique, Seuil, Paris, 1991.

EUROBAROMETRE, Les Européens et la sûreté nucléaire, 2007, http://ec.europa.eu/public_opinion/archives/ebs/ebs_271_fr.pdf 
Entre gestion de crise et vigilance...

FROSH P., PINCHEVSKI A., "Crisis-Readiness and Media Witnessing”, Communication Review, 2009, 12/3, p. 295-304.

GAMSON W.A., MODIGLIANI A., "Media discourse and public opinion on nuclear power : a constructionist approach", American Journal of Sociology, 1989, vol.95, $\mathrm{N}^{\circ} 1, \mathrm{p} .1-37$.

GIDDENS A., The consequences of modernity, Cambridge University Press, Cambridge, 1990.

GILPIN D.R., "Narrating the organizational self: Reframing the role of the news release", Public Relations Review, 2008, 34/1, p. 9-18.

GODARD O., HENRY C., LAGADEC P., MICHEL-KERJAN E., Traité des nouveaux risques, Gallimard, 2002.

HOLLADAY S.J., "Crisis Communication Strategies in the Media Coverage of Chemical Accidents", Journal of Public Relations Research, 2009, 35/2, p. 208-217.

HOLMES B., HENRICH N., HANCOCK S., LESTOU V., "Communicating with the public during health crises: experts' experiences and opinions", Journal of Risk Research, 2009, 12/6, p. 793-807.

HUPET P., «La communication dans le domaine agro-alimentaire : l'indispensable contradiction ", HUPET P., dir., Risques et systèmes complexes : les enjeux de la communication, Bruxelles, P.I.E.-Peter Lang, 2001, p.137-154.

LAGADEC P., La Gestion des crises, McGraw Hill, 1991.

MULLER P., «Esquisse d'une théorie du changement dans l'action publique. Structures, Acteurs et cadres cognitifs », Revue Française de Science politique, 2005, vol. 55, $\mathrm{N}^{\circ} 1, \mathrm{p} .155-187$.

SEON-KYOUNG A., GOWER K.K., "How do the news media frame crises? A content analysis of crisis news coverage", Public Relation Review, 2009, 35/2, p. 107-112.

SLOVIC P., "Perceived risk, trust and democracy", Risk Analysis, 1993, vol.13, pp. 675-682.

SUREL Y., "The role of cognitive and normative frames in policymaking", Journal of European Public Policy, 2000, 7/4, p. 495-512. 